\title{
Effectiveness and Safety of Mechanical Thrombectomy with Stent Retrievers in Basilar Artery Occlusion : Comparison with Anterior Circulation Occlusions
}

\author{
Soo Young Hu, M.D., Ho Jun Yi, M.D., Dong Hoon Lee, M.D., Jae Taek Hong, M.D., Jae Hoon Sung, M.D., Sang Won Lee, M.D. \\ Department of Neurosurgery, St. Vincent's Hospital, College of Medicine, The Catholic University of Korea, Suwon, Korea
}

Objective : Acute basilar artery occlusion (BAO) is associated with severe neurological dysfunction and high mortality rates. The benefits of mechanical thrombectomy in BAO have not been explored in recent clinical trials. Therefore, we analyzed outcomes of stent retriever mechanical thrombectomy for $\mathrm{BAO}$, and compared with anterior circulation occlusions (ACO).

Methods : In total, 161 consecutive patients (24 BAO, 137 ACO) who underwent mechanical thrombectomy with the stent retriever between January 2013 and August 2016 enrolled in our study. All patients underwent clinical assessment with the National Institutes of Health Stroke Scale (NIHSS) and modified Rankin scale (mRS). Radiological results were used to evaluate thrombolysis in cerebral infarction (TICl) scores and successful recanalization was defined by $\mathrm{TICl} 2 \mathrm{~b}$ or 3.

Results : Mean NIHSS scores at 24 hours and 30 days were significantly higher in the BAO group than the ACO group ( $p=0.021$, $p=0.001)$. mRS at 90 days was significantly higher in the BAO group (4.2) compared with the ACO group (3.0) $(p=0.003)$. The BAO group (2.0) performed fewer stent retriever passages than the ACO group (2.7) $(p=0.049)$. There were no significant differences between the two groups in terms of complications, but the BAO patients experienced a higher mortality (16.6\%) rate than ACO patients (5.8\%) ( $p=0.001)$. In subgroup analysis of $\mathrm{BAO}$, patients with short procedure times achieved successful recanalization ( $p=0.001)$ and successfully recanalized patients exhibited more favorable $\mathrm{mRS}$ at 90 days $(p=0.027)$.

Conclusion : In our study, mechanical thrombectomy of BAO patients showed worse clinical outcome and higher mortality rate than ACO patients. However, mechanical thrombectomy with a stent retriever in BAO is an effective treatment, because successfully recanalized patients showed good clinical outcome in BAO patients.

Key Words : Basilar artery $\cdot$ Stroke $\cdot$ Stents $\cdot$ Thrombectomy.

- Received : April 17, 2017 •Revised : June 4, 2017 •Accepted : August 2, 2017

- Address for reprints : Ho Jun Yi, M.D.

Department of Neurosurgery, St. Vincent's Hospital, College of Medicine, The Catholic University of Korea, 93 Jungbu-daero, Paldal-gu, Suwon 16247, Korea Tel : +82-31-249-8176, Fax : +82-31-2649-7020, E-mail : 431anarchy@naver.com

This is an Open Access article distributed under the terms of the Creative Commons Attribution Non-Commercial License (http://creativecommons.org/licenses/by-nc/4.0) which permits unrestricted non-commercial use, distribution, and reproduction in any medium, provided the original work is properly cited. 


\section{INTRODUCTION}

Acute basilar artery occlusion (BAO) is rare but is known to be related to severe neurologic dysfunction and morbidity or mortality ${ }^{2,23)}$. Early recanalization is a key goal of acute ischemic stroke therapy for BAO and anterior circulation occlusion $(\mathrm{ACO})^{9,16,20)}$. However, even with recanalization, the natural course and prognosis of ischemic stroke caused by $\mathrm{BAO}$ is worse compared with that of $\mathrm{ACO}^{3)}$. Several studies have reported that $>80 \%$ of the BAO patients with an initial severe deficit had an unfavorable outcome, and poor prognosis strongly correlated with failure to achieve recanalization of the $\mathrm{BAO}^{14,15)}$.

Since 2015, multiple randomized controlled trials (RCTs) have consistently reported that endovascular mechanical thrombectomy is clearly beneficial in treating ACO acute ischemic stroke ${ }^{4,5,10)}$; however, studies investigating mechanical thrombectomy of ischemic stroke caused by BAO are limited. Several trials have introduced treatment methods for BAO, such as intravenous thrombolysis with tissue-plasminogen activator (t-PA), intra-arterial thrombolysis and mechanical thrombectomy and combination of these ${ }^{7,11,26)}$. However, it remains unclear what is the most effective treatment protocol for patients with $\mathrm{BAO}^{8)}$. Recently, the most widely used mechanical thrombectomy is a stent retriever technique with the Solitaire stent (ev3, Irvine, CA, USA) or Trevo stent (Stryker Neurovascular, Fremont, CA, USA). These self-expanding and fully retrievable stents enable recanalization rates of up to $90 \%$ in patients with ischemic stroke caused by $\mathrm{ACO}^{13,21)}$. However, studies focusing on the safety and effectiveness of mechanical thrombectomy for acute ischemic stroke caused by BAO remain limited.

Therefore, we compared the effectiveness and safety of stent retriever mechanical thrombectomy for BAO with ACO. In addition, we investigated the clinical and radiologic outcomes of stent retriever mechanical thrombectomy for acute ischemic stroke caused by BAO and analyzed the variables influencing factors associated with arterial recanalization and functional outcomes.

\section{MATERIALS AND METHODS}

\section{Study population}

Institutional review board approval was obtained before starting this retrospective study (IRB No. VC17RISI0036). A total of 161 patients with acute ischemic stroke who underwent stent retrieval thrombectomy at our institution between January 2013 and August 2016 were enrolled. The authors divided the patients into two groups : mechanical thrombectomy for ACO (ACO group) and mechanical thrombectomy for BAO (BAO group). Eligible patients had an occlusion of the internal carotid artery (ICA), middle cerebral artery (M1 or M2), anterior cerebral artery (ACA), or basilar artery, as established by computed tomography angiography (CTA) or perfusion computed tomography (CT) with magnetic resonance image (MRI) diffusion weighted images, and a score of $\geq 2$ on the National Institutes of Health Stroke Scale (NIHSS; range, $0-42$, with higher scores indicating more severe neurologic deficits). Patients who received intravenous (IV) alteplase ( $\mathrm{t}$ PA) within 4.5 hours after stroke onset with a maximum dose of $90 \mathrm{mg} / \mathrm{kg}$ were enrolled. Basilar artery occlusion was divided into three categories by starting point of occlusion (Proximal, from fusion of the vertebral arteries to anterior inferior cerebellar arteries; mid, from anterior inferior cerebellar arteries to superior cerebellar artery; distal, from superior cerebellar artery to the division into posterior cerebral arteries). In subgroup analysis, the authors re-divided the BAO patients according to clinical outcomes and recanalization. Inclusion criteria were as follows : 1) acute ischemic stroke due to intracranial artery occlusion confirmed by CTA or volume perfusion CT; 2) neurologic dysfunction; 3) diffusion-perfusion mismatch revealed at volume perfusion CT and diffusion MRI; and 4) $\leq 8$ hours elapsed from stroke onset for ACO and $\leq 12$ hours for BAO. Exclusion criteria were as follows : 1 ) hemorrhage in the CT; 2) large ischemic core : $\geq 6$ Alberta Stroke Program Early CT Score score; 3) patients who had contraindications on MRI or contrast-enhanced CT; and 4) patients who underwent mechanical thrombectomy with non-stent retrieval or other devices (such as Eric, Penumbra or chemical).

\section{Thrombectomy procedure}

Mechanical treatment involved thrombus retraction with a retrievable stent (Solitaire FR : $4 \times 20 \mathrm{~mm}$ and $6 \times 30 \mathrm{~mm}$ or Trevo XP : $4 \times 20 \mathrm{~mm}$ and $6 \times 25 \mathrm{~mm}$ ) and in some cases an added balloon-guiding catheter (BGC) (Merci; Concentric Medical Inc., Mountain View, CA, USA). Merci 8 French catheter was used in ACO and Merci 7 French catheter was used in BAO. 


\section{Outcomes and complications}

Multimodal factors were reviewed, including patient age, sex, underlying disease, location of occluded vessel, IV t-PA, and BGC. All patients underwent clinical assessment with NIHSS at baseline, after 24 hours, and at 30 days, and modified Rankin Scale (mRS) score at 90 days by the review of medical records. Good clinical outcomes were defined as $\geq 2 \mathrm{mRS}$ at 90 days. Differences were analyzed in multiple time intervals : time from symptom onset to groin puncture time, procedure time (from groin puncture to reperfusion time), and time from onset to reperfusion. Radiologic results were evaluated by the number of stent passages, thrombolysis in cerebral infarction (TICI) score ${ }^{25)}$ and successful recanalization, which was defined as a TICI score of $2 b$ to 3 . Safety variables and complications were analyzed regarding distal emboli, symptomatic hemorrhage, and mortality within 90 days. In subgroup analysis of BAO patients, patients were divided into two groups according to the successful recanalization and clinical outcomes and then multiple categories were evaluated. The length of thrombus for BAO patients was measured at digital subtraction angiography (DSA) images and it was defined as the length of the basilar artery not contrasted on the DSA. Two neuroradiologists (Y. K. Ihn and W. S. Jung) were unaware of the treatment-group assignments and evaluated all neuroimaging studies.

\section{Statistical analysis}

$\chi^{2}$ test or Fisher's exact test were used to analyze differences in multi-variable factors between the two groups. MannWhitney U test or Student's t-test were employed to evaluate differences in clinical and radiological variables between the two groups. And $\chi^{2}$ test or Fisher's exact test and MannWhitney U test or Student's t-test were used for subgroup analysis of BAO patients. Two-tailed $p$-values of $\leq 0.05$ were considered to indicate a significant difference. Data were analyzed using SPSS ver. 18 statistical software (South Wacker Drive, Chicago, IL, USA).

\section{RESULTS}

\section{Baseline characteristics}

A total of 161 patients were enrolled between January 2013 and August 2016 from a single institution. The locations of BAO were as follows : distal ( $n=7 ; 29.1 \%)$, mid $(n=7 ; 29.1 \%)$, and proximal $(\mathrm{n}=10 ; 41.7 \%)$, of these cases, there were 2 cases in the mid occlusion accompanied the distal occlusion, and 1 case in the proximal occlusion involves the mid occlusion and 2 cases in the proximal occlusion involves distal occlusion. And ACO infarct locations were : middle cerebral artery $(\mathrm{n}=94 ; 68.7 \%)$, ICA $(\mathrm{n}=42 ; 30.6 \%)$, and ACA $(\mathrm{n}=1 ; 0.7 \%)$. Mean age of patients with $\mathrm{BAO}$ was 65.7 years, compared with 65.5 in the ACO group. BAO group contained 24 patients (13 men, 11 women) and the ACO group contained 137 patients (78 men, 59 women). Multi-variable factors, such as sex, age, hypertension, diabetes mellitus, atrial fibrillation, coronary artery disease, dyslipidemia, and smoking were not significantly different between the groups. A total of 73 patients $(45.3 \%)$ received IV alteplase (t-PA), and there were no statistically significant differences between the groups $(p=0.506)$. The proportion of applied BGC was significantly lower in the BAO group (17.5\%) than the ACO group (51.8\%) $(p=0.001$; Table 1). In terms of time interval, the time interval from onset to groin puncture in the BAO group (268 minutes) was significantly longer than in the ACO group (216 minutes) ( $p=0.018$ ). Similarly, the time interval from onset to reperfusion was significantly longer in the BAO group (390 minutes) than in the ACO group (333 minutes) $(p=0.039)$. However, there were no statistically significant differences in procedure time between the two groups ( $\mathrm{BAO}: \mathrm{ACO}=122: 116 ; p=0.271$ ) (Table 1).

\section{Clinical and radiologic outcomes and complica- tions}

In terms of clinical outcomes, the mean initial NIHSS score was higher in BAO group with statistically significance (BAO : $\mathrm{ACO}=14.2$ : 10.4). The mean NIHSS scores at 24 hours were significantly higher in the BAO group (9.6) than in the ACO group (7.1) $(p=0.021)$ and the mean NIHSS score at 30 days was significantly higher in the BAO group (8.6) than for ACO (5.3) $(p=0.001)$. The mRS at 90 days in the BAO group (4.2) was significantly higher $(p=0.003)$, compared with that of the ACO group (3.0). The number of stent passages was significantly lower in the BAO group (2.0) than in the ACO group (2.7) $(p=0.049)$. There was no statistically significant difference in rate of successful recanalization between the two groups in terms of radiologic outcome (BAO : $\mathrm{ACO}=79.1 \%$ : 
$80.2 \% ; p=0.899)$. In terms of prognosis, the BAO group $(16.6 \%)$ exhibited a significantly higher mortality rate than the ACO group (5.8\%) ( $p=0.001)$. When comparing complications, there were no significant differences in distal emboli
( $\mathrm{BAO}: \mathrm{ACO}=33.3 \%: 19.7 \% ; p=0.178)$ and symptomatic hemorrhage (BAO : $\mathrm{ACO}=4.1 \%: 8.7 \% ; p=0.129)$ between the two groups (Table 2).

Table 1. Baseline characteristics

\begin{tabular}{|c|c|c|c|c|}
\hline & Total & Basilar artery occlusion & Anterior circulation occlusion & $p$-value* \\
\hline Total & 161 & 24 & 137 & \\
\hline \multirow[t]{3}{*}{ Location } & & Distal : 7 (29.1) & MCA : 94 (68.7) & \\
\hline & & Mid : 7 (29.1) & ICA : 42 (30.6) & \\
\hline & & Prox. : 10 (41.7) & ACA : $1(0.7)$ & \\
\hline $\operatorname{Sex}(M: F)$ & $91: 70$ & $13: 11$ & $78: 59$ & 0.826 \\
\hline Mean age & $65.5(22-87)$ & $65.7(32-85)$ & $65.5(22-87)$ & 0.929 \\
\hline HTN & $97(60.2)$ & $13(60.2)$ & $84(61.3)$ & 0.652 \\
\hline DM & $44(27.3)$ & $7(19.7)$ & $37(27.0)$ & 0.827 \\
\hline Af. & $70(43.4)$ & $5(36.2)$ & $65(47.4)$ & $0.024 \dagger$ \\
\hline CAD & $5(3.1)$ & $1(20.8)$ & $4(2.9)$ & 0.745 \\
\hline Dyslipidemia & $66(40.9)$ & $8(33.3)$ & $58(42.3)$ & 0.400 \\
\hline Smoking & $51(31.6)$ & $9(17.5)$ & $42(30.6)$ & 0.635 \\
\hline$t-P A$ & $73(45.3)$ & $9(34.0)$ & $64(46.7)$ & 0.506 \\
\hline$B G C$ & $73(45.3)$ & $2(17.5)$ & 71 (51.8) & $0.001^{\dagger}$ \\
\hline Stent $(S: T)$ & $161(99: 62)$ & $24(14: 10)$ & $137(85: 52)$ & 0.821 \\
\hline $\begin{array}{l}\text { Time from onset to groin puncture } \\
\text { (minutes) }\end{array}$ & $224(36-612)$ & $268(59-552)$ & $216(36-612)$ & $0.018^{\dagger}$ \\
\hline Procedure time (minutes) & $117(27-360)$ & $122(40-285)$ & $116(27-360)$ & 0.271 \\
\hline Time from onset to reperfusion (minutes) & $341(90-870)$ & $390(137-675)$ & $333(90-870)$ & $0.039^{\dagger}$ \\
\hline
\end{tabular}

Values are presented as number (\%) or number (interquartile range) unless otherwise indicated. ${ }^{*} p$-values are calculated by $\chi^{2}$ test or Fisher's exact test and Mann-Whitney U test or Student's t-test. 'Statistically significant. MCA : middle cerebral artery, ICA : internal carotid artery, Prox : proximal, ACA : anterior cerebral artery, $\mathrm{M}$ : male, $\mathrm{F}$ : female, HTN : hypertension, DM : diabetes mellitus, Af. : atrial fibrillation, CAD : coronary artery disease, t-PA tissue-plasminogen activator, BGC : ballon guiding catheter, $\mathrm{S}$ : solitaire, $\mathrm{T}$ : trevo

Table 2. Outcomes and safety variables

\begin{tabular}{lcccc}
\hline & Total & Basilar artery occlusion & Anterior circulation occlusion & p-value* \\
\hline NIHSS score at initial & $11.0(2-34)$ & $14.2(2-34)$ & $10.4(3-26)$ & $0.028^{\dagger}$ \\
NIHSS score at 24 hours & $7.4(0-28)$ & $9.6(2-28)$ & $7.1(2-20)$ & $0.021^{\dagger}$ \\
NIHSS score at 30 days & $5.7(0-25)$ & $8.6(0-25)$ & $3.0(0-6)$ & $0.001^{\dagger}$ \\
mRS at 90 days & $3.2(0-6)$ & $4.2(0-6)$ & $2.7(1-10)$ & $0.003^{\dagger}$ \\
No of passes & $2.6(1-10)$ & $2.0(1-6)$ & $110(80.2)$ & $0.049^{\dagger}$ \\
TICl score of 2b or 3 on final angiogram & $129(80.1)$ & $19(79.1)$ & $27(19.7)$ & 0.899 \\
Distal emboli & $35(21.7)$ & $8(33.3)$ & $12(8.7)$ & $8(5.8)$ \\
Symptomatic hemorrhage & $13(8.0)$ & $1(4.1)$ & $4(16.6)$ & 0.178 \\
Mortality & $12(7.4)$ & 0.129 & $0.001^{\dagger}$ \\
\hline
\end{tabular}

Values are presented as number (\%) or number (interquartile range) unless otherwise indicated. ${ }^{*} p$-values are calculated by $\chi^{2}$ test or Fisher's exact test and Mann-Whitney U test or Student's t-test. ${ }^{\dagger}$ Statistically significant. NIHSS : National Institutes of Health Stroke Scale, mRS : modified Rankin Scale, $\mathrm{TICl}$ : thrombolysis in cerebral infarction 
Usefulness of Mechanical Thrombectomy for Basilar Artery Occlusion I Hu SY, et al.

\section{Subgroup analysis of BAO patients}

The authors performed the subgroup analysis of $24 \mathrm{BAO}$ group patients, successfully recanalized patients was 19 (79.1\%) and good clinical outcome achieved patients was 10 (41.7\%). The successful recanalization was co-related with short procedure time, shorter time interval from symptom onset to reperfusion and good mRS at 90 days with statically significance ( $p=0.001, p=0.036, p=0.019$ ). In addition, short thrombus length was significantly related with successful recanalization ( $p=0.008$ ), but other factors (sex, age, underlying disease, t-PA, initial NIHSS score, and number of stent passage) were not significantly co-related with successful recanalization (Table 3). In terms of clinical outcomes, the authors defined that the patients with 90 days mRS 2 or more as a good clinical outcome. This study shows that shorter procedure time, lower initial NIHSS score and successful recanalization were read to good clinical outcomes with statical sig- nificance ( $p=0.004, p=0.018, p=0.027$ ) and shorter thrombus length was significantly related with good clinical outcomes $(p=0.011)$. There was no significant correlation between other factors and good clinical outcomes (Table 4).

\section{DISCUSSION}

It is widely known that ischemic stroke caused by $\mathrm{BAO}$ is associated with high mortality rates, severe disability, and good clinical outcomes after recanalization. Several studies recommended that mechanical thrombectomy in acute ischemic stroke caused by $\mathrm{BAO}$ within the first $6-8$ hours may be reasonable ${ }^{19,27)}$. However, the RCTs investigating revascularization therapies of $\mathrm{BAO}$ are rare and most studies are performed retrospectively, in which the effectiveness of mechanical thrombectomy for $\mathrm{BAO}$ compared with $\mathrm{ACO}$ is ascertained.

Table 3. Subgroup analysis : classification with successful recanalization of basilar artery occlusion

\begin{tabular}{|c|c|c|c|}
\hline & $\begin{array}{l}\text { Successfully recanalized } \\
(\mathrm{TICl}=2 \mathrm{~b} \text { or } 3)\end{array}$ & $\begin{array}{l}\text { Non-successfully recanalized } \\
(\mathrm{TICl}=0,1 \text { or } 2 \mathrm{a})\end{array}$ & $p$-value* \\
\hline Total & $19(79.1)$ & $5(20.9)$ & \\
\hline $\operatorname{Sex}(M: F)$ & $11: 8$ & $2: 3$ & 0.630 \\
\hline Mean age & $66.3(32-85)$ & $63.4(49-81)$ & 0.674 \\
\hline HTN & $8(42.1)$ & $5(100)$ & 0.401 \\
\hline DM & $5(26.3)$ & $2(40.0)$ & 0.608 \\
\hline Af. & $5(26.3)$ & $0(0.0)$ & 0.316 \\
\hline Dyslipidemia & $6(31.5)$ & $2(40.0)$ & 0.772 \\
\hline $\mathrm{t}-\mathrm{PA}$ & $7(36.8)$ & $2(40.0)$ & 0.897 \\
\hline Smoking & $7(36.8)$ & $2(40.0)$ & 0.897 \\
\hline Stent $(\mathrm{S}: \mathrm{T})$ & $19(12: 7)$ & $5(2: 3)$ & 0.615 \\
\hline Time from onset to groin puncture (minutes) & $216(36-612)$ & $255(192-346)$ & 0.781 \\
\hline Procedure time (minutes) & $116(27-360)$ & $213(153-285)$ & $0.001^{\dagger}$ \\
\hline Time from onset to reperfusion (minutes) & $333(90-870)$ & $468(421-520)$ & $0.036^{\dagger}$ \\
\hline Initial NIHSS score & $13.9(2-21)$ & $15.3(6-34)$ & 0.599 \\
\hline NIHSS score at 24 hours & $9.8(1-28)$ & $8.6(4-17)$ & 0.727 \\
\hline NIHSS score at 30 days & $8.3(0-5)$ & $9.6(3-15)$ & 0.720 \\
\hline mRS at 90 days & $3.9(0-6)$ & $5.2(3-6)$ & $0.019^{\dagger}$ \\
\hline No of passes & $1.9(1-6)$ & $2.2(1-3)$ & 0.659 \\
\hline Thrombus length (minutes) & $10.5(4.1-25.1)$ & $30.6(14.1-39.1)$ & $0.008^{\dagger}$ \\
\hline
\end{tabular}

Values are presented as number (\%) or number (interquartile range) unless otherwise indicated. ${ }^{*} p$-values are calculated by $\chi^{2}$ test or Fisher's exact test and Mann-Whitney U test or Student's t-test. ${ }^{\top}$ Statistically significant. TICI : thrombolysis in cerebral infarction, M : male, F : female, HTN : hypertension, DM : diabetes mellitus, Af. : atrial fibrillation, t-PA : tissue-plasminogen activator, $\mathrm{S}:$ solitaire, T : trevo, NIHSS : National Institutes of Health Stroke Scale, mRS : modified Rankin Scale 
Table 4. Subgroup analysis : classification with clinical outcomes of basilar artery occlusion

\begin{tabular}{|c|c|c|c|}
\hline & Good outcome ( 90 days $m R S \leq 2$ ) & Poor outome (90 days $m R S \geq 3$ ) & $p$-value* \\
\hline Total & $10(41.7)$ & $14(58.3)$ & \\
\hline $\operatorname{Sex}(M: F)$ & $6: 4$ & $7: 7$ & 0.386 \\
\hline Mean age & $58.5(32-79)$ & $63.4(49-85)$ & 0.075 \\
\hline HTN & $3(30.0)$ & $10(71.4)$ & 0.182 \\
\hline DM & $2(20.0)$ & $4(28.5)$ & 0.384 \\
\hline Af. & $1(10.0)$ & $4(28.5)$ & 0.608 \\
\hline Dyslipidemia & $5(50.0)$ & $3(21.4)$ & 0.307 \\
\hline$t-P A$ & $3(30.0)$ & $6(42.8)$ & 0.669 \\
\hline Smoking & $4(40.0)$ & $5(35.7)$ & 0.356 \\
\hline Stent $(S: T)$ & $10(5: 5)$ & $14(9: 5)$ & 0.653 \\
\hline Time from onset to groin puncture (minutes) & $235(59-420)$ & $285(135-552)$ & 0.529 \\
\hline Procedure time (minutes) & $63(40-90)$ & $151(49-285)$ & $0.004^{\dagger}$ \\
\hline Time from onset to reperfusion (minutes) & $299(137-490)$ & $436(230-675)$ & 0.054 \\
\hline No of passes & $1.5(1-3)$ & $2.2(1-6)$ & 0.374 \\
\hline Initial NIHSS score & $11.1(2-20)$ & $16.4(5-34)$ & $0.018^{\dagger}$ \\
\hline $\mathrm{TICl}$ score of $2 \mathrm{~b}$ or 3 on final angiogram & $10(100)$ & $9(64.2)$ & $0.027^{\dagger}$ \\
\hline Thrombus length (minutes) & $9.8(4.1-22.2)$ & $28.6(9.1-39.1)$ & $0.011^{\dagger}$ \\
\hline
\end{tabular}

Values are presented as number (\%) or number (interquartile range) unless otherwise indicated. ${ }^{*} p$-values are calculated by $\chi^{2}$ test or Fisher's exact test and Mann-Whitney U test or Student's t-test. 'Statistically significant. mRS : modified Rankin Scale, M : male, F : female, HTN : hypertension, DM : diabetes mellitus, Af. : atrial fibrillation, t-PA : tissue-plasminogen activator, S : solitaire, T : trevo, NIHSS : National Institutes of Health Stroke Scale, TICI : thrombolysis in cerebral infarction

Therefore, this study focused on the effectiveness and safety of mechanical thrombectomy in BAO.

There were no significant differences in sex, age, medical history, t-PA, smoking, and stent type between $\mathrm{BAO}$ and $\mathrm{ACO}$ patients in this study. The present data suggested that mechanical thrombectomy in patients with $\mathrm{BAO}$ achieved an acceptable successful recanalization rate (79.1\%). These values are superior to those of previous studies investigating intravenous thrombolysis $(65-68 \%)^{22,24)}$, and are not low when compared to other studies that performed mechanical thrombectomy after BAO $(75-81 \%)^{1,9)}$. Mechanical thrombectomy in BAO (79.1\%) exhibited a similar rate of successful recanalization when compared to ACO (80.2\%) in our study. When comparing the mean thrombectomy time between the stents used in our series, there was little difference between BAO (122 minutes) and ACO (116 minutes), although the mean number of stent passages was lower in patients with $\mathrm{BAO}(2.0)$, compared to those with ACO (2.7). These results are probably due to the increased trial-and-error associated with $\mathrm{BAO}$ in the process of approaching the lesion in addition to a stent re- triever. However, these radiologic outcomes of mechanical thrombectomy with a stent retriever in $\mathrm{BAO}$ are not dissimilar from those of ACO. These results indicated the technical feasibility and applicability of mechanical thrombectomy using stent retrieval to treat $\mathrm{BAO}$.

However, BAO patients exhibited poorer clinical outcomes than ACO as evident in all clinical outcome values (NIHSS at 24 hours, 30 days, and mRS at 90 days) and these results are not significantly different from previously published articles ${ }^{1)}$. Although there is a little difference in successful recanalization rate, but there is a more difference in clinical outcomes, these results is may be derived from poor initial NIHSS score and delayed time to treatment of BAO. In our study, the mean initial NIHSS score of BAO (14.2) was slightly higher than that of ACO (10.4) and the time elapsed from onset to groin puncture and from onset to reperfusion was significantly longer for BAO compared with ACO. In terms of prognosis, mortality rates were significantly higher in the BAO group than for ACO. This finding resulted in poor clinical outcomes and a severe initial neurologic deficit of $\mathrm{BAO}$, which is consistent 
Usefulness of Mechanical Thrombectomy for Basilar Artery Occlusion I Hu SY, et al.

Table 5. Review of preiovusly reported articles of mechanical thrombectomy for BAO

\begin{tabular}{|c|c|c|c|c|c|c|}
\hline Source & $\begin{array}{c}\text { Number of } \\
\text { patients }\end{array}$ & $\begin{array}{c}\text { Time window } \\
\text { (hour) }\end{array}$ & $\begin{array}{c}\text { Successful } \\
\text { recanalization (\%) }\end{array}$ & $\begin{array}{l}\text { Good clinical } \\
\text { outcomes (\%) }\end{array}$ & ICH (\%) & Mortality (\%) \\
\hline This study & 24 & 10.2 & 79.1 & 41.7 & 4.1 & 16.6 \\
\hline Gilberti et al. & 32 & 15.0 & 87.5 & 40.6 & 6.3 & 25.0 \\
\hline Huo et al. ${ }^{12)}$ & 36 & 12.0 & 94.5 & 27.8 & 16.7 & 30.6 \\
\hline Park et al. ${ }^{18)}$ & 16 & 5.8 & 81 & 56 & 0 & 6 \\
\hline Costalat et al. ${ }^{6}$ & 16 & 24.0 & 81 & 44 & 2 & 25 \\
\hline Alonso de Leciñana et al." & 52 & 12.0 & 75 & 40 & 2 & 33 \\
\hline
\end{tabular}

$\mathrm{ICH}$ : intracerebral hemorrhage

with previous studies ${ }^{23,26)}$. When comparing complications, distal embolization frequency was higher with BAO, but symptomatic hemorrhage occurred less in BAO than ACO. This increased incidence of distal embolization with $\mathrm{BAO}$ may be associated with a low application rate of $\mathrm{BGC}$ for $\mathrm{BAO}$ compared with $\mathrm{ACO}^{17}$. The use of $\mathrm{BGC}$ was limited due to the narrow diameter and tortuosity of the vertebra-basilar artery (17.5\%), compared with anterior circulation (51.8\%), therefore, distal embolization increased during mechanical thrombectomy of BAO than ACO.

In, subgroup analysis of $\mathrm{BAO}$, our study revealed that the pateints with successful recanalization were 19 (79.1\%) and good clinical outcome were 10 (41.7\%). Although the standards are slightly different for each article, our results are not far behind compared with previously reported articles ${ }^{1,6,8,12,18)}$ (Table 5). Especially in our study, the patients who achieved successful recanalization were related with shorter procedure time and good 90 days mRS score than non-successfully recanalized patients. Patients with 90 days mRS less than 2 were considered to be achieved clinically good outcome, shorter procedure time, lower initial NIHSS score and successful recanalization on final angiography were co-related with good clinical outcome. In addition, shorter length of basilar artery thrombus was an independent predictor for successful recanalization and good clinical outcome in our study. These findings are thought to be related that the length of thrombus in BAO may correlate with obstruction of basilar paramedian perforating arteries ${ }^{8)}$. Therefore, the extension of subsequent thrombus on basilar artery lead to broad brainstem damage, so long thrombus in basilar artery make a brainstem ischemia that cannot be recovered even with successful recanalization. Other factors were not associated successful recanalization and good clinical outcome with statistically significance. Other studies suggest that prognosis-related factors of BAO include severity of initial neurologic status, age, time interval to treatment and successful recanalization ${ }^{28,29)}$. In our study, shorter procedure time, successful recanalization, shorter thrombus length and good clinical outcomes were correlated, but there was no statistically significant difference detected for age.

Although our study revealed that mechanical thrombectomy with a stent retriever in $\mathrm{BAO}$ was related to poorer clinical outcomes and higher mortality rate than ACO, these findings seem to support the notion that mechanical thrombectomy with the stent retrievers is key to the revascularization of BAO. This aspect may have previously been questioned due to the associated treatment in previous studies (intravenous thrombolysis and intra-arterial urokinase, or other retrievers, such as the Penumbra or the Merci retriever) and is congruent with the efficacy that thrombectomy with stent retrievers has shown in the treatment of other large-vessel occlusions.

The present study has limitations : 1) We evaluated patients with a retrospective design and were not able to conduct a RCT, and poorly designed non-RCTs are more likely to suffer from various types of bias; 2) this study had a relatively small sample size, as the sample was taken from a single center; 3 ) the difference in the number of patients between $\mathrm{BAO}$ and ACO was too great; and 4) the choice of devices was affected by the preference of the neuro-interventionists and was not standardized. Other potential unmeasured confounding variables were not controlled for, although every effort was made to adjust for the possibility of spurious results. Despite these limitations, our study demonstrated the effectiveness and safety of mechanical thrombectomy with a stent retriever for BAO. 


\section{CONCLUSION}

In our study, mechanical thrombectomy of BAO patients showed worse clinical outcome and higher mortality rate than ACO patients. However, BAO subgroup analysis revealed that a short procedure time and successful recanalization were correlated with good clinical outcomes; therefore, mechanical thrombectomy with a stent retriever in acute ischemic stroke caused by BAO may be an effective treatment for clinical and radiologic outcomes. Through future RCTs, the safety and effectiveness of mechanical thrombectomy in acute ischemic stroke caused by BAO will be fully elucidated.

\section{References}

1. Alonso de Leciñana $M$, Kawiorski MM, Ximénez-Carrillo Á, CruzCulebras A, García-Pastor A, Martínez-Sánchez P, et al. : Mechanical thrombectomy for basilar artery thrombosis : a comparison of outcomes with anterior circulation occlusions. J Neurointerv Surg, 2016 [Epub ahead of print]

2. Archer $C R$, Horenstein $S$ : Basilar artery occlusion: clinical and radiological correlation. Stroke $8: 383-390,1977$

3. Arnold M, Nedeltchev K, Schroth G, Baumgartner RW, Remonda L, Loher TJ, et al. : Clinical and radiological predictors of recanalisation and outcome of 40 patients with acute basilar artery occlusion treated with intra-arterial thrombolysis. J Neurol Neurosurg Psychiatry 75 : 857 862,2004

4. Berkhemer OA, Fransen PS, Beumer D, van den Berg LA, Lingsma HF, Yoo AJ, et al. : A randomized trial of intraarterial treatment for acute ischemic stroke. N Engl J Med 372 : 11-20, 2015

5. Campbell BC, Mitchell PJ, Kleinig TJ, Dewey HM, Churilov L, Yassi N, et al. : Endovascular therapy for ischemic stroke with perfusion-imaging selection. N Engl J Med 372 : 1009-1018, 2015

6. Costalat V, Machi P, Lobotesis K, Maldonado I, Vendrell JF, Riquelme $C$, et al. : Rescue, combined, and stand-alone thrombectomy in the management of large vessel occlusion stroke using the solitaire device: a prospective 50-patient single-center study: timing, safety, and efficacy. Stroke 42 : 1929-1935, 2011

7. Eckert B, Koch C, Thomalla G, Kucinski T, Grzyska U, Roether J, et al. : Aggressive therapy with intravenous abciximab and intra-arterial rtPA and additional PTA/stenting improves clinical outcome in acute vertebrobasilar occlusion: combined local fibrinolysis and intravenous abciximab in acute vertebrobasilar stroke treatment (FAST): results of a multicenter study. Stroke 36 : 1160-1165, 2005

8. Gilberti N, Gamba M, Premi E, Costa A, Vergani V, Delrio I, et al. : Endovascular mechanical thrombectomy in basilar artery occlusion: variables affecting recanalization and outcome. J Neurol 263 : 707-713, 2016
9. Gory B, Eldesouky I, Sivan-Hoffmann R, Rabilloud M, Ong E, Riva R, et al. : Outcomes of stent retriever thrombectomy in basilar artery occlusion: an observational study and systematic review. J Neurol Neurosurg Psychiatry 87 : 520-525, 2016

10. Goyal M, Demchuk AM, Menon BK, Eesa M, Rempel JL, Thornton J, et al. : Randomized assessment of rapid endovascular treatment of ischemic stroke. N Engl J Med 372 : 1019-1030, 2015

11. Hacke W, Zeumer H, Ferbert A, Brückmann H, del Zoppo GJ : Intraarterial thrombolytic therapy improves outcome in patients with acute vertebrobasilar occlusive disease. Stroke 19 : 1216-1222, 1988

12. Huo X, Gao F, Sun X, Ma N, Song L, Mo D, et al. : Endovascular mechanical thrombectomy with the solitaire device for the treatment of acute basilar artery occlusion. World Neurosurg 89 : 301-308, 2016

13. Kabbasch C, Mpotsaris A, Chang DH, Hiß S, Dorn F, Behme D, et al. : Mechanical thrombectomy with the trevo provue device in ischemic stroke patients: does improved visibility translate into a clinical benefit? J Neurointerv Surg $8: 778-782,2016$

14. Lindsberg PJ, Mattle HP : Therapy of basilar artery occlusion: a systematic analysis comparing intra-arterial and intravenous thrombolysis. Stroke 37 : 922-928, 2006

15. Lindsberg PJ, Pekkola J, Strbian D, Sairanen T, Mattle HP, Schroth G : Time window for recanalization in basilar artery occlusion: speculative synthesis. Neurology 85 : 1806-1815, 2015

16. Mazighi M, Serfaty JM, Labreuche J, Laissy JP, Meseguer E, Lavallée PC, et al. : Comparison of intravenous alteplase with a combined intravenous-endovascular approach in patients with stroke and confirmed arterial occlusion (RECANALISE study): a prospective cohort study. Lancet Neurol 8 : 802-809, 2009

17. Oh JS, Yoon SM, Shim JJ, Doh JW, Bae HG, Lee KS : Efficacy of balloonguiding catheter for mechanical thrombectomy in patients with anterior circulation ischemic stroke. J Korean Neurosurg Soc 60 : 155-164 2017

18. Park BS, Kang CW, Kwon HJ, Choi SW, Kim SH, Koh HS, et al. : Endovascular mechanical thrombectomy in basilar artery occlusion: initial experience. J Cerebrovasc Endovasc Neurosurg 15 : 137-144, 2013

19. Powers WJ, Derdeyn CP, Biller J, Coffey CS, Hoh BL, Jauch EC, et al. : 2015 American heart association/american stroke association focused update of the 2013 guidelines for the early management of patients with acute ischemic stroke regarding endovascular treatment: a guideline for healthcare professionals from the american heart association/american stroke association. Stroke 46 : 3020-3035, 2015

20. Rha JH, Saver $\mathrm{JL}:$ The impact of recanalization on ischemic stroke outcome: a meta-analysis. Stroke 38 : 967-973, 2007

21. Roth C, Papanagiotou P, Behnke S, Walter S, Haass A, Becker C, et al. : Stent-assisted mechanical recanalization for treatment of acute intracerebral artery occlusions. Stroke 41 : 2559-2567, 2010

22. Sairanen $T$, Strbian D, Soinne $L$, Silvennoinen $H$, Salonen $O$, Artto V, et al. : Intravenous thrombolysis of basilar artery occlusion: predictors of recanalization and outcome. Stroke 42 : 2175-2179, 2011

23. Schonewille WJ, Wijman CA, Michel P, Rueckert CM, Weimar C, Mattle $\mathrm{HP}$, et al. : Treatment and outcomes of acute basilar artery occlusion in 
Usefulness of Mechanical Thrombectomy for Basilar Artery Occlusion | Hu SY, et al.

the basilar artery international cooperation study (BASICS): a prospective registry study. Lancet Neurol 8 : 724-730, 2009

24. Strbian D, Sairanen T, Silvennoinen $H$, Salonen 0 , Kaste M, Lindsberg PJ : Thrombolysis of basilar artery occlusion: impact of baseline ischemia and time. Ann Neurol 73 : 688-694, 2013

25. Suh SH, Cloft HJ, Fugate JE, Rabinstein AA, Liebeskind DS, Kallmes DF : Clarifying differences among thrombolysis in cerebral infarction scale variants: is the artery half open or half closed? Stroke $44: 1166-1168$, 2013

26. van Houwelingen RC, Luijckx GJ, Mazuri A, Bokkers RP, Eshghi OS, Uyttenboogaart $M$ : Safety and outcome of intra-arterial treatment for basilar artery occlusion. JAMA Neurol 73 : 1225-1230, 2016
27. Wahlgren N, Moreira T, Michel P, Steiner T, Jansen O, Cognard C, et al. : Mechanical thrombectomy in acute ischemic stroke: consensus statement by ESO-karolinska stroke update 2014/2015, supported by ESO, ESMINT, ESNR and EAN. Int J Stroke 11 : 134-147, 2016

28. Zeumer H, Freitag HJ, Grzyska U, Neunzig HP : Local intraarterial fibrinolysis in acute vertebrobasilar occlusion. Technical developments and recent results. Neuroradiology 31 : 336-340, 1989

29. Zeumer H, Freitag HJ, Zanella F, Thie A, Arning C : Local intra-arterial fibrinolytic therapy in patients with stroke: urokinase versus recombinant tissue plasminogen activator (r-TPA). Neuroradiology 35 : 159-162, 1993 